\title{
XIII. On spectra arising from the dissociation of water vapour and the presence of dark lines in gaseous spectra
}

\author{
John Trowbridge
}

To cite this article: John Trowbridge (1902) XIII. On spectra arising from the dissociation of water vapour and the presence of dark lines in gaseous spectra, Philosophical Magazine Series 6, 4:19, 156-161, DOI: 10.1080/14786440209462826

To link to this article: http://dx.doi.org/10.1080/14786440209462826

曲 Published online: 15 Apr 2009.

Submit your article to this journal $[\pi$

Џll Article views: 2

Q View related articles $\longleftarrow$

Citing articles: 1 View citing articles $\square$ 
of single atoms, the work of $\$ \S 27,28$ remains valid; and thus we arrive at the very important and interesting conclusion that when there is repulsion between nearest nets, attraction between next-nearests, and no force between next-nextnearests or any farther, the disturbance from homogeneousness in the neighbourhood of the bounding plane consists in alternate diminutions and augmentations of density becoming less and less as we travel inwards, but remaining sensible at distances from the boundary amounting to several times the distance from net to net.

XIII. On Spectra arising from the Dissociation of Water Vapour and the Presence of Dark Lines in Gaseous Spectra. By JoHn Trowbridge *.

\section{[Plate III.]}

IN passing from the study of the light emitted by gases under the effect of electrical discharges to the investigation of the light produced by discharges of great quantity, one enters a new field of research. In previous papers on the spectra of hydrogen I have stated my convictions of the importance of the rôle played by water-vapour in glass spectrum-tubes. The results of further study emphasize these convictions. With powerful discharges in hydrogen, oxygen, and rarefied air, even when these gases are dried with the ntmost care, I always obtain the same spectrum, which I regard as that arising from the dissociation of water-vapour which is always present in glass tubes. The bright-line spectrum, moreover, at high temperatures is accompanied by a faint continuous spectrum on which are dark lines which indicate a selective reversibility in the silver salt. This reversibility, it seems to me, is of great significance in the application of photography to astrophysics.

It has long been recognized that spectrum analysis is an extremely delicate method of recognizing the presence of a gas or the vapour of a metal under the excitation of heat; and when the improvements in photography enabled us to obtain permanent records of the spectra of gases, it was supposed that we had a means of escaping from the fallacies of eye-observations which arose from personal idiosyncracies. If the photographic plate were a perfect instrument for recording the infinite number of vibrations which light can communicate to atoms of matter, we should certainly feel that we had made a great advance in physical science. When

* Communicated by the Author. 
we reflect, however, on the supposition that emulsions containing silver salts are capable of responding and giving a permanent record of all waves of light, even in the portion of the spectrum considered most actinic, when the waves exceed a certain intensity, we are conscious that we rely without proof upon an infinite range of photochemical action; and indeed I show in this paper the existence of a selective reversibility produced on the photographic plate by powerful discharges producing light of great intensity.

Realizing the importance of studying the behaviour of gases under different forms of excitation, I have collected in the rooms devoted to spectrum analysis in this laboratory three forms of apparatus : an induction-coil actuated by a very efficient liquid break, giving a spark of 30 inches in air; a step-up transformer, excited by an alternating current, producing with glass condensers of about $\cdot 3$ microfarad discharges of an inch in length of great body; and a storagebattery of twenty thousand cells. A plant of this nature I conceive to be necessary in the present stage of spectrum analysis ; for molecular motions excited in rarefied gases vary greatly with the kind of electrical discharge. In the application of photography to spectrum analysis one is immediately confronted with the necessity of submitting the gas to a comparatively long electrical stimulus in order to obtain a negative. Even with a concave grating of short focus several discharges are necessary with a narrow slit. Each discharge is capable of modifying the condition of the gas. This fact is well recognized by taking successive photographs upon the same plate with different strengths of current. A simple form of plate-holder enables this to be done. One obtains a striking example of the instability of a spectrum-tube filled, apparently, with dry hydrogen when one subjects it first to very powerful discharges from a glass condenser of 6 microfarad charged by a storage-battery of twenty thousand cells, with practically no self-induction in the circuit, and follows this excitation by an alternating current of mach less quantity. The powerful discharge gives what I term the spectrum arising from the dissociation of water-vapour; and the alternating current gives the spectra of argon. This results, I suppose, from the oxidization of traces of air in the tube under the action of the dissociation of the water-vapour. The presence of hydrogen is concealed. On cooling, the tube again shows the four-line spectrum of hydrogen. The period of the condenser-discharges which I have employed varied from one five-hundred thousandth of a second to one millionth. The practically instantaneous current, therefore, varied from five 
thousand amperes to ten thousand. The revolving-mirror method showed that the pilot spark was mainly effective, and that the subsidiary oscillations were feeble. The spectrumtube speedily became milk-white from the sodium set free from the glass. Lord Rayleigh has shown how to demonstrate the presence of argon from very small quantities of air (Phil. Mag. [6] vol. i. p. 108, 1901). My method is substantially his, except that I employ very powerful discharges which sèt free a sufficient amount of sodium-vapour from the glass: and the oxygen is supplied from the dissociation of watervapour which is always in evidence when powerful discharges are employed. The production of argon under these circumstances I regard as a striking proof that $I$ am dealing in this investigation with the spectra arising from the dissociation of water-vapour. From the same tube one can, by modifying. the strength and character of the electrical discharges, obtain what is generally termed the four-line spectrum of hydrogen, the spectrum of sodium, the spectra of argon, and the spectrum arising from the dissociation of water-vapour. Doubtless one could recognize also the spectrum of helium; I am not yet sufficiently familiar with it.

In the course of the stindy of the water-rapour spectrum one is naturally led to photograph the spectrum of the electric spark under water. It is possible to obtain powerful discharges of any suitable length under distilled water by inclosing the spark terminals in glass tubes, allowing only a small portion of the platinum terminals to project from the ends of the tubes. If the terminals are immersed more than one inch under the water, the resulting explosion is apt to break the glass containing-vessel. The light of these discharges under distilled water is white and extraordinarily brilliant to the eye. When it is examined by the spectroscope one sees a continuous spectrum; and one obtains a continuous spectrum by photography even in the most actinic portion of the spectrum. On bringing the spark terminals to the surface of the water, one immediately obtains the four-line spectrum ot hydrogen or water-vapour. To what is due the continuous spectrum under water? Does it result from the production of the dissociation-spectrum of water-vapour under great pressure? That there is great pressure is shown by the sudden explosion, which is sufficient to blow the small tamping of water out of both ends of the containing tube. If the water is covered with a thin tilm of oil, this oil is immediately disseminated through the water, making a milky white emulsion which remains for days.

When we turn to powerful discharges through Plücker or 
Geissler tubes filled with hydrogen which has been dried with care, we also obtain a faint continuous spectrum on which are bright lines and dark lines. Moreover, what are apparently the strongest bright lines of the dissociationspectrum of water-vapour are not reversed. There is a selective reversibility which arises at high temperatures.

This fact seems to me of great importance in the application of photography to the study of celestial phenomena. Reversal of spectrum-lines does not necessarily indicate reversing layers of cooler gases, and in certain cases may arise from photochemical action of the silver salt. One immediately thinks in this comnexion of the phenomenon of dark lightning or the Clayden effect, and of the interesting experiments of Professor Nipher*. Spectrum analysis, however, reveals a selective reversibility which must be carefully studied before we can properly interpret the records of photography. There are doubtless many states of vibration, even in the actinic portion of the spectrum, which are not recorded by the silver salt : for this selective reversibility may obliterate or prevent a permanent record. I have obtained this reversing action with different emulsions on glass and also on celluloid films. The strongest reversals are approximately at wave-lengths $4227,3930,3965$. There is also a faint reversal at wave-length 3953 . Reversals are often seen on the negative which disappear in the fixing-bath.

In this investigation ten thousand cells were employed to charge a glass condenser of 6 microfarad. The charge was sent through Geissler tubes with practically no self-induction in the discharge-circuit. The bore of the capillary tubes was $1 \mathrm{~mm}$, and the tubes were filled with apparently dry hydrogen at a pressure of approximately $\cdot 1 \mathrm{~mm}$. The tubes were also filled with oxygen and also with rarefied air at the same pressure. I have reached a limit in subjecting glass tubes to powerful discharges, and am now turning my attention to obtaining quartz tubes in the hope of securing a more resisting material.

In a previous paper $\dagger$ I expressed my conviction that the four-line spectrum observed in the protuberances of the sun is an evidence of the presence of water-vapour in the sun's atmosphere, and an evidence, therefore, of the presence of oxygen. In the spectrum (Plate III. fig. 7, B) one sees on the negative two reversed lines which coincide with the great $\mathrm{H}, \mathrm{H}$ lines of the solar spectrum. These are seen bright in

* "On Certain Properties of Light-Struck Plates," Trans. Academy of St. Louis, Mo. U. S. vol. x. No. 6 .

† Phil. Mag. [5] vol. l. p. 338 (1900). 
$\mathrm{B}$, fig. 4. One also sees a strong reversed line at approximately wave-length 4227; and there is a reversed band coinciding with the solar region of reversed lines between wave-lengths 4315-4285. These regions in the sun are doubtless composite photographs of reversals of many elements. I believe that there is a basis of reversal due to the dissociation of water-vapour.

The nomenclature of the stars in regard to their types of spectra may need revision. The higher temperature I get the more dark lines I obtain.

An excess of dissociation of water-vapour may suffice to give at a comparatively low temperature the bright-line spectrum of hydrogen. At higher temperature the dissociation of this vapour in the presence of atmospheric air may give dark lines. The intense light due to the dissociation of water-vapour under the effect of powerful discharges is the nearest approach to sunlight which $I$ have been able to produce. Its actinic effect is greater than that of magnesium, zinc, or aluminium. It may be that the variability of certain stars is due to a variability in the amount of water-vapour which is being dissociated; and one is led to conjecture whether the light of the sun's atmosphere may not be due to an electrical dissociation. The selective reversibility of the silver salts seems to me, therefore, of great interest in the subject of astrophysics; for we can have reversible effects on the photographic plates which are not due to the reversing effect of colder layers of gases. In other words, we have actions recorded which are on the plates and not in the heavens. The intense light due to the dissociation of watervapour may entirely mask the fainter light of the metallic lines in stars which show only gaseous spectra, especially when we consider the varying distances of the stars. I have employed electrodes of platinum, copper, silver, aluminium, iron, and found no trace of the lines of their vapour in the spectrum of the dissociation of water-vapour. Even when caustic soda is present in the tubes, although it fills the tube with a brilliant yellow light with comparatively low discharges, no trace is seen of it when the tube is excited with powerful discharges. Then we have the brilliant white light of the water-vapour spectrum.

The silver salt, therefore, does not respond to all rates of vibration; or if it does respond, the molecular action is unstable and there is no resultant reaction which is evidenced by a photographic image. There may be spectra at very high instantaneous temperatures which we cannot photograph. It 
seems reasonable to suppose that the silver mulecule is limited in its rate of vibrations, and that the photographic plate as well as the human eye is a limited instrument of research.

On Plate III., A represents the normal solar spectrum in the neighbourhood of the great H, H lines. B represents the gaseous spectra. The photographs were taken with a Rowland concave grating, and are not enlarged or touched in any way. Unfortunately the reproductions do not give many of the reversals, and some of the bright lines do not appear. This; is especially the case in fig. 5 . Figs. 1 and $2, \mathrm{~B}$, are spectrit of oxygen and traces of rarefied air taken with comparatively low enrrent and voltage at a pressure of 1 millimetre. Fig. 3 is a spectrum of hydrogen under the same conditions. Fig. 4 is the spectrum arising from the dissociation of water-vipour with very powerful discharges. Fig. 5 shows a line of selective reversibility at wave-length 4227 . On the negative the two brilliant lines (fig. 4) which closely coincide with the great $\mathrm{H}, \mathrm{H}$ lines of the solar spectrum are seen to be reversed, and therefore appear as dark lines. This reversal is shown in fig. 7. The gaseous spectrum B of fig. 7 elosely resembles in general features the solar spectrum photographed with a wide slit in order to give the general distribution of darkness and light.

My conclusions are as follows:-

Dissociation of water-vapour takes place in the atmosphere of the sun. Oxygen, therefore, must be present. From a carefnl study of my negatives I regard the evidence for the presence of this vapour as conclusive as that generally accepted for the presence of sodium in the sun.

The dissociation of water-vapour by powerful electric discharges in the presence of small amounts of atmospheric air results in. the production of argon even in tubes presumably filled with hydrogen.

Dark lines occur in the spectra of gases which increase with the intensity of the light, and are photochemical in their origin.

The great brilliancy of the dissociation spectrum of watervapour, which obscures the presence of metallic spectra, and the presence of dark lines due to photochemical reversals, makes one cautious in accepting photographic evidence in regard to the states of development of stars.

Phil. Mag. S. c. Vol. 4. No. 19. July 1902. 
Phil. Mag. S. 6. Vol. 4. Pl. III.

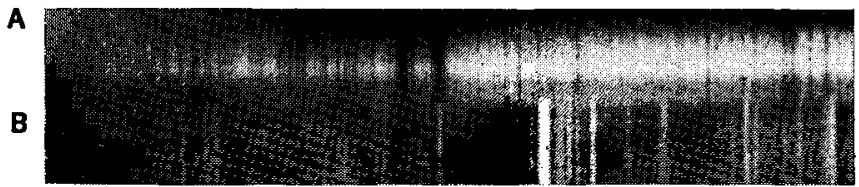

Fig. 1.

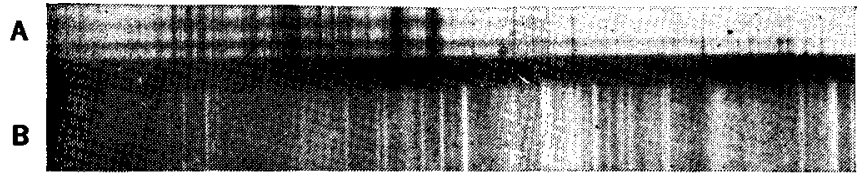

FIG. 2.

B

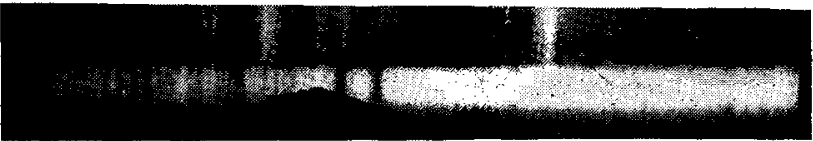

Fia. 3.

A

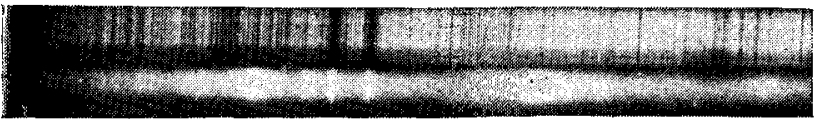

Fig. 4.

B

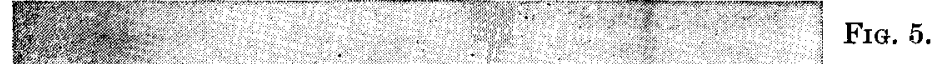

B

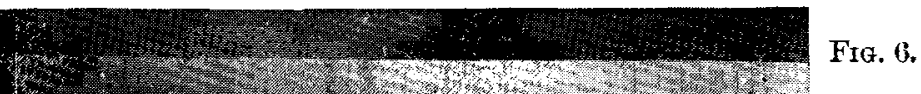

A 